\title{
A LARGE-SCALE CO IMAGING OF THE GALACTIC CENTER WITH THE NOBEYAMA 45-M TELESCOPE: SHELL STATISTICS AND STAR FORMATION HISTORY
}

\author{
TETSUO HASEGAWA \\ Institute of Astronomy, The University of Tokyo \\ Osawa, Mitaka, Tokyo 181, Japan \\ TOMOHARU OKA \\ The Institute of Physical and Chemical Research (RIKEN) \\ Hirosawa, Wako, Saitama 351-01, Japan \\ FUMIO SATO \\ Department of Astronomy and Earth Sciences, Tokyo Gakugei \\ University \\ Nukui-kita machi, Koganei, Tokyo 184, Japan \\ AND \\ MASATO TSUBOI AND ATSUSHI YAMAZAKI \\ Institute of Astrophysics and Planetary Sciences, Ibaraki University \\ Bunkyo, Mito, Ibaraki 310, Japan
}

\section{Shell Statistics}

As a key program of the 45-m telescope at Nobeyama Radio Observatory, we have made high-resolution $\mathrm{CO}$ images of the Galactic center region with the $2 \times 2$ focal plane array receiver. The data consist of $44,000{ }^{12} \mathrm{CO}$ and $13,000{ }^{13} \mathrm{CO}$ spectra taken with $16^{\prime \prime}$ beams spaced by 34 ". The ${ }^{12} \mathrm{CO}$ image covers roughly $-1.5^{\circ} \leq l \leq+3.4^{\circ}$ and $-0.6^{\circ} \leq b \leq+0.6^{\circ}$ (Oka et al. 1997b)

In the data we find a number of features that were not known before; among them are numerous shells, arched filaments, circular holes, and their mixtures. As the first attempt, we have selected an area $-0.37^{\circ} \leq l \leq$ $+0.55^{\circ}$ and identified a total of 412 such structures which we collectively call "shells". Identification was done by eye mainly on a sequence of $5 \mathrm{~km} \mathrm{~s}^{-1}$ wide velocity channel diagrams of ${ }^{12} \mathrm{CO}$ while we changed the intensity 
level continuously. Many of them are identified in more than two adjacent velocity channels, and $\sim 10 \%$ show the kinematic signatures of expanding shells. Their radii range from a few parsecs to a few tens; $75 \%$ of them are smaller than $5 \mathrm{pc}$ in radius, while $10 \%$ are larger than $10 \mathrm{pc}$. Their rims are not complete in most cases, being discernible only in two to three quarters on the average.

If we extrapolate the count in the central $140 \mathrm{pc}$ to the entire $500 \mathrm{pc}$ extent of the Galactic center molecular cloud complex, there may be $\sim 10^{3}$ or more shells. Their volume filling factor in the $500 \times 500 \times 50 \mathrm{pc}^{3}$ of molecular gas layer is $\sim 0.2$, corresponding to $\sim 20$ shells to be found along any line of sight through it. The abundance of expanding shells may naturally cause the high pressure of the interstellar matter there (e.g., Spergel \& Blitz 1992; Oka et al. 1997a).

An average shell with a radius $R=6$ pc has a mass $M \approx 3 \times 10^{4} M_{\odot}$. Its kinetic energy is $E_{\mathrm{k}} \approx\left(M \sigma_{\mathrm{v}}^{2} / 2\right)=1 \times 10^{50} \mathrm{ergs}$, and should be younger than $\left(R / \sigma_{\mathrm{v}}\right)=5 \times 10^{5}$ years. These parameters suggest that they are supernova

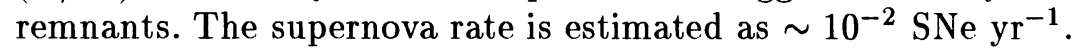

\section{Star Formation History}

These shells, if they are confirmed to be SNRs of Type II, are fossil records of the past history of star formation. A standard IMF expects that the number of luminous $O$ stars $\left(\geq 20 M_{\odot}\right)$ formed in a unit time is $1 / 5$ times that of the progenitors of Type II SNe $\left(\geq 8 M_{\odot}\right)$. The latter should be equal to the SN rate estimated above if the star formation has been steady for the last $(\mathrm{a}$ few $) \times 10^{7}$ years. Then, we expect $\sim 10^{4}$ luminous $O$ stars in the main sequence $\left(\tau_{\mathrm{MS}} \leq 1 \times 10^{7}\right.$ years $)$. In contrast, the number of observed luminous $\mathrm{O}$ stars in the central $500 \mathrm{pc}$ of the Galaxy is much smaller $(\sim 100)$.

This strongly suggests that the star formation rate has decreased by a large factor in the last $\sim(1-2) \times 10^{7}$ years. The Galactic center region may be in a post-starburst phase.

\section{References}

Oka, T., Hasegawa, T., Hayashi, M., Handa, T., \& Sakamoto, S. (1997a) CO (J=2-1) line observations of the Galactic center molecular cloud complex. II. Dynamical structure and physical conditions, $A p J$ in press.

Oka, T., Hasegawa, T., Sato, F., Tsuboi, M., \& Miyazaki, A. (1997b) A large-scale CO imaging of the Galactic center, $A p J S$, submitted

Spergel, N., \& Blitz, L. (1992) Extreme gas pressures in the Galactic bulge, Nature, 357, pp. $665-666$ 